\title{
ATIVIDADE FOTODINÂMICA E CONCEITOS: UM EXPERIMENTO DEMONSTRATIVO
}

\author{
Ana Claudia Pedrozo da Silva ${ }^{a}$, Camila Fabiano de Freitas ${ }^{a}$, André Luiz Tessaro ${ }^{b}$, Wilker Caetano ${ }^{a}$, Silvana Maria de \\ Oliveira Santin $^{\mathrm{a}, *}$, Noboru Hioka ${ }^{\mathrm{a}}$ e Graciana Freitas Palioto ${ }^{\mathrm{b}}$ \\ aDepartamento de Química, Universidade Estadual de Maringá, 87020-900 Maringá - PR, Brasil \\ bUniversidade Tecnológica Federal do Paraná, Apucarana - PR, Brasil
}

Recebido em 14/11/2017; aceito em 27/02/2018; publicado na web em 19/03/2018

\begin{abstract}
PHOTODYNAMIC ACTIVITY AND CONCEPTS: A DEMONSTRATIVE EXPERIMENT. A simple and low cost experiment is proposed to chemistry students associating the Photodynamic Activity involving Chemical and Biochemical concepts. The inclusion of this experiment in undergraduate courses in Chemistry promotes interdisciplinarity and contextualization, combining current and relevant topics to the teaching of program content. The experiment used the Methylene Blue and Erythrosine B as dyes to demonstrate the photodynamic action of these photosensitizers in the inhibition of microbial growth. Both dyes presented satisfactory results against environmental microorganisms. The growth inhibition was caused exclusively by the photochemical processes undergone by the dye/light/oxygen, with LED light exposure.
\end{abstract}

Keywords: photodynamic therapy; methylene blue; erythrosine B, LED, inactivation of microorganism.

\section{INTRODUÇÃO}

Ao longo dos séculos a luz vem sendo empregada na medicina, tendo registros datados há mais de 4000 anos no Egito, na Grécia e na Índia, nos quais a luz solar era empregada na cura de vários distúrbios. Pensava-se que somente a luz solar era responsável pela cura de doenças como vitiligo, psoríase, raquitismo e até psicose. Com o avanço científico notou-se que geralmente o efeito era obtido após a ingestão de plantas, seguida pela exposição solar. ${ }^{1}$ Ressalta-se que naquele momento, não se conhecia a existência de raios ultravioleta (UV), que foram descobertos após $1801 .^{2}$

Anos mais tarde pesquisadores perceberam que o efeito terapêutico estava relacionado à combinação da luz solar com os pigmentos presentes nas plantas ingeridas, ou seja, aos corantes. A idéia de usar um corante como agente terapêutico surgiu de uma observação realizada por Oscar Raab, na época aluno do professor von Tappeiner no Instituto Farmacológico do Ludwig Maximilian, na Universidade de Munique (1897-1898). Raab realizava um estudo sobre a letalidade do vermelho de acridina sobre o protozoário causador da malária (Paramecium caudatum). Ele identificou letalidade reduzida em um dia chuvoso, entretanto, em um dia ensolarado, o efeito foi pronunciado. A partir desta observação, ele concluiu que a luz ativava o corante, induzindo o microrganismo à morte. Em 1903, é reportada a primeira utilização fotodinâmica de corantes no tratamento de tumores por von Tappeiner e Jesionek, utilizando o corante eosina. ${ }^{3}$

A partir de então, inúmeros estudos foram realizados com intuito de compreender os efeitos fotodinâmicos acarretados pela combinação da luz com os corantes. Descobriu-se que a atividade só era efetiva se a luz empregada tivesse emissão em comprimentos de onda condizente com o espectro de absorção dos corantes, processo fotoquímico primário, e se houvesse a presença de oxigênio molecular $\left({ }^{3} \mathrm{O}_{2}\right)$. Assim surgiu a base da chamada Terapia Fotodinâmica (TFD), envolvendo a ativação de um composto fotossensibilizador (FS, corante) por luz de comprimento de onda adequado na presença de oxigênio. Dessa interação resulta a geração de oxigênio singleto $\left({ }^{1} \mathrm{O}_{2}\right)$ e espécies reativas de oxigênio (EROs), que são responsáveis pela morte de células in situ. ${ }^{3,4}$

\footnotetext{
*e-mail: smoliveira@uem.br
}

Após a irradiação do fotossensibilizador no tecido biológico alvo, o mesmo é promovido a um estado singleto excitado ( $\left.{ }^{1} \mathrm{FS} *\right)$, podendo então sofrer mudança em sua multiplicidade de spin através do cruzamento inter-sistemas, levando-o ao estado tripleto excitado ( $\left.{ }^{3} \mathrm{FS} *\right)$. O estado tripleto possui maior tempo de vida, o que faz com que reações fotoquímicas importantes geralmente ocorram nesse estado. Tendo o cromóforo chegado à ${ }^{3} \mathrm{FS} *$, dois mecanismos são propostos para o processo que leva à geração de espécies citotóxicas. No mecanismo Tipo I, ocorre a transferência de elétrons entre o ${ }^{3} \mathrm{FS} *$ e substratos biológicos, gerando íons-radicais que tendem a reagir com o oxigênio formando as chamadas espécies reativas de oxigênio, como o ânion superóxido $\left(\mathrm{O}_{2}^{-}\right)$e o radical hidroxila $\left(\mathrm{OH}^{*}\right)$. Já no mecanismo Tipo II, ocorre a transferência de energia do ${ }^{3} \mathrm{FS} *$ diretamente para o oxigênio em seu estado fundamental, que já é tripleto $\left({ }^{3} \mathrm{O}_{2}\right)$, levando à formação de oxigênio singleto $\left({ }^{1} \mathrm{O}_{2}\right)$, espécie altamente oxidante. Ambos os caminhos podem levar à morte celular, no entanto, o mecanismo Tipo II é proposto como principal via de ação da TFD. ${ }^{5}$

A partir da década de 50, com os estudos de Schwartz e Lipson para tratamento do câncer de mama, o desenvolvimento dos primeiros medicamentos foi iniciado. ${ }^{1} \mathrm{Na}$ década de 70 , os trabalhos de Dougherty sobre derivados hematoporfirínicos culminaram no desenvolvimento do princípio ativo Photofrin ${ }^{\circledR}$, o primeiro FS com uso aprovado pelos órgãos reguladores, fato ocorrido somente no ano de $1998 .^{5-7}$ A continuidade dos estudos levou ao desenvolvimento dos medicamentos da chamada segunda geração, com dois novos fármacos aprovados pela agência americana FDA (Food and Drug Administration) em 1999: o Levulan ${ }^{\circledR}$ Kerastick, uma pró-droga para lesões de pele, e o Visudyne ${ }^{\circledast}$ para degeneração macular da retina, cujo princípio fotoativo é uma benzoporfirina.

A potencialidade da técnica na Inativação Fotodinâmica de Microrganismos (IFDMO) ou Terapia Fotodinâmica Antimicrobiana (TFDA), em bactérias, fungos e vírus já é conhecida há muitos anos. ${ }^{8-}$ ${ }^{10}$ No entanto, o desenvolvimento de drogas antimicrobianas, como a penicilina (durante a II Guerra Mundial), praticamente inutilizou fotossensibilizadores como ativos antimicrobianos. ${ }^{9,11} \mathrm{O}$ atual destaque da IFDMO (ou TFDA) se deve principalmente à disseminação da AIDS e do aumento de casos de infecções hospitalares e bactérias resistentes a antibióticos. ${ }^{12} \mathrm{~A}$ técnica oferece vantagens como um amplo espectro de ação, sendo eficiente inclusive sobre microrganismos 
resistentes a antibióticos e apresentando menores efeitos colaterais nocivos quando comparada a outros tratamentos. ${ }^{8,12,13}$ Além disso, uma vez que a inativação se dá por meio da produção de espécies altamente reativas com ataque a múltiplos alvos celulares, não se considera possível o desenvolvimento de resistência microbiana. Por essas vantagens, a utilização da TFDA tem se mostrado relevante, por exemplo, na esterilização de bolsas de sangue, na periodontia e no tratamento e conservação de alimentos. ${ }^{12}$

Hoje, os tratamentos fotodinâmicos são utilizados nos EUA, Canadá, Japão, Europa entre outros. No Brasil, ainda que tímido, emprega-se o tratamento em centros oncológicos como o Hospital Amaral Carvalho de Jaú, ${ }^{14}$ diversos centros oftalmológicos no tratamento da degeneração macular da retina, em centros odontológicos para eliminação de bactérias relacionadas à infecções endodônticas e periodontais, ${ }^{15}$ no tratamento contra cáries ${ }^{16} \mathrm{e} \mathrm{em}$ implantes dentários. ${ }^{17} \mathrm{~A}$ TFDA também já é utilizada em tratamentos dermatológicos para doenças de pele como as ceratoses actínicas e carcinomas basocelulares ${ }^{18}$ e no tratamento de úlceras em pacientes diabéticos. ${ }^{19,20}$

Quanto a escolha dos corantes FS para utilização em TFD, deve ser baseada em suas características físico-químicas, como alta absorção de luz e geração de ${ }^{1} \mathrm{O}_{2}$. Além disso, é imprescindível que o FS apresente farmacocinética favorável, baixa toxicidade no escuro, elevada estabilidade, fácil obtenção e preferencialmente baixo custo. ${ }^{21}$ A solubilidade do FS em meio biológico é outro fator crucial, já que os processos de autoagregação do FS diminuem consideravelmente a geração de ${ }^{1} \mathrm{O}_{2}$ devido à processos de auto-colisões. ${ }^{5,7}$

Dentre os fotossensibilizadores que detém essas características, pode-se destacar o corante catiônico Azul de Metileno (AM, Figura 1A). O AM é um corante da classe dos fenotiazínicos muito utilizado na área microbiológica e comercializado como antisséptico. Sem a presença de luz também vem sendo utilizado na área médica no tratamento da metahemoglobinemia e tem despertado interesse como potencial princípio ativo no tratamento da doença de Alzheimer. ${ }^{22,23}$

Para a TFD, o Azul de Metileno é um interessante fotossensibilizador devido sua alta absorção de luz na região do vermelho $\left(\varepsilon_{664}=39,0 \times 10^{3} \mathrm{~L} \mathrm{~mol}^{-1} \mathrm{~cm}^{-1}\right)^{24}$ e um bom rendimento quântico de oxigênio singleto $\left(\Phi_{\Delta}{ }^{1} \mathrm{O}_{2}=0,50\right)$ em água. ${ }^{25} \mathrm{Em}$ termos de aplicação fotodinâmica, o AM tem sido extensivamente reportado na literatura em tratamentos de casos de melanomas, ${ }^{26}$ células tumorais cerebrais ${ }^{27}$ e células de rim. ${ }^{28}$ Para a TFDA, o AM também se mostrou eficaz contra bactérias resistentes ${ }^{29,30}$ e no tratamento de doenças negligenciadas como a Leishmaniose cutânea ${ }^{31}$ e casos de osteomielite em pacientes diabéticos. ${ }^{32}$

Outro FS altamente promissor é o corante xantênico Eritrosina B (ERI), cuja estrutura é representada na Figura 1B. Em condições de $\mathrm{pH}$ fisiológico $(\sim 7,4)$, a ERI encontra-se na forma dianiônica apresentando elevado rendimento quântico de oxigênio singleto $\left(\phi_{\Delta}{ }^{1} \mathrm{O}_{2}=0,63\right)$ e alta absortividade molar na região do verde $\left(\varepsilon_{532}=96,6 \times 10^{3} \mathrm{~L} \mathrm{~mol}^{-1} \mathrm{~cm}^{-1}\right)$ em água. ${ }^{33,34}$ Este composto possui a vantagem de ser aprovado para o uso na ausência de luz em alimentos e em tratamentos odontológicos como evidenciador da placa bacteriana. A ERI/Luz tem sido testada com sucesso em tratamentos do biofilme dental via TFDA contra o microrganismos Streptococus mutans. ${ }^{35,36}$ Estudos recentes também demonstraram efeitos significativos da ERI associada à luz na inibição de patógenos alimentares. ${ }^{37}$

Outro aspecto crucial em tratamentos fotodinâmicos é a escolha correta da fonte de luz. Essa seleção deve ser feita com base na região de absorção do FS e no tipo de lesão a ser tratada. A fonte mais utilizada ainda é o LASER, devido sua elevada potência, e sua luz pode ser dirigida ao local da lesão por meio de fibras óticas. No entanto, o LASER é extremamente custoso e a pequena dimensão de seu foco
(A)

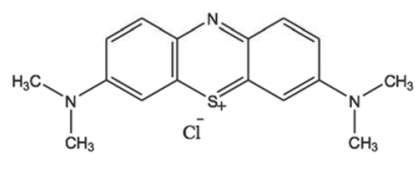

Azul de Metileno
(B)

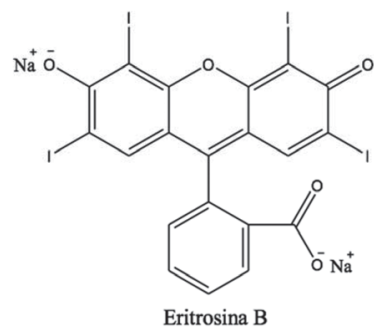

Figura 1. Estruturas dos fotossensibilizadores (A) Azul de Metileno e (B) Eritrosina $B$

de luz é restritiva a lesões menores. Este vem sendo substituído por alternativas muito mais econômicas, como os diodos emissores de luz (LED, do inglês, Light Emitting Diode), ${ }^{3}$ sendo que um conjunto de unidades LED permite irradiar lesões de áreas grandes.

Enfatiza-se que a fotoexcitação exige a sobreposição entre o espectro de absorção do FS e o espectro de emissão da fonte de luz (processo fotoquímico primário). Assim, quanto maior a sobreposição espectral e a irradiância (potência por área) da luz, maior será o número de fótons absorvidos pelo FS e maior a geração de espécies citotóxicas, acentuando a morte celular. ${ }^{38}$

Toxicidade mínima em tecidos normais, efeitos sistêmicos negligenciáveis, morbidade reduzida ao longo prazo e o não aparecimento de resistência tumoral e microbiana fazem da TFD/TFDA uma opção terapêutica valiosa. Além de todos esses benefícios, o procedimento ainda envolve uma quantidade significativa de conceitos físico-químicos, fotofísicos e biológicos que podem e devem ser abordados no ensino. A necessidade por práticas de ensino que envolvam conceitos multidisciplinares é latente e o número de publicações nesse contexto tem crescido significativamente. ${ }^{39-41}$ Contudo, no que concerne à técnica fotodinâmica, seu uso para esse fim é escasso. Em cursos de Química a TFD é descrita em exemplos de reações fotoquímicas em tópicos de Cinética Química e Espectroscopia, como no livro de Físico-Química dos autores Peter Atkins e Julio de Paula. ${ }^{42}$ No campo de pesquisas, vários grupos nas pós-graduações em Química, Física, Odontologia, Farmácia, Bioquímica, Zootecnia, Agronomia, Veterinária, Medicina, Fisiologia, entre outros, inclusive no Brasil, têm investigado os mais diferentes fotossensibilizadores, com diversas modificações em suas formulações e técnicas de aplicação, voltados a diferentes patologias. No sentido de abordar o elevado potencial da TFD/ TFDA em tratamentos oncológicos e microbiológicos, e também visando o estreitamento entre o ensino de graduação com pesquisas científicas de ponta, esse trabalho demonstra a possibilidade de contextualização da terapia por meio de um experimento simples e de baixo custo. O experimento pode ser aplicado em cursos de nível Superior de Química e áreas correlatas, relacionando a aplicação de reações fotoquímicas na TFDA para esterilização de materiais e tratamentos biomédicos.

Para a realização dos experimentos, amostras de microrganismos foram obtidas do ambiente. Escolheram-se dois fotossensibilizadores para análise quantitativa do experimento, o Azul de Metileno e a Eritrosina B, ambos de fácil obtenção e baixo custo, iluminados com dispositivos de LED vermelho e verde, respectivamente.

Para uma abordagem efetiva, sugere-se a leitura preliminar dos artigos: "Terapia Fotodinâmica: princípios, potencial de aplicação e perspectivas" do autor Antônio Eduardo da Hora Machado; 3 "Terapia Fotodinâmica: aspectos farmacológicos, aplicações e avanços recentes no desenvolvimento de medicamentos" dos autores Símplicio, Maionchi e Hioka; ${ }^{4} \mathrm{e}$ "Inativação Fotodinânica de microrganismos" de Janice Perussi, ${ }^{12}$ nestes casos, todos artigos publicados na revista Química Nova visando o Ensino de Química no Brasil. 


\section{PARTE EXPERIMENTAL}

\section{Materiais}

O Azul de Metileno é facilmente adquirido em casas farmacêuticas na forma de solução aquosa $1 \%$ (empresa ADV). A Eritrosina B é igualmente de fácil aquisição na forma de corante alimentício líquido da marca Mix de cor "rosa cereja", adquirido em mercados assim como o caldo de carne. Ágar-ágar foi adquirido da Ingá Alimentos Produtos Naturais na forma de pó levemente amarelado vendido em embalagens de $50 \mathrm{~g}$, e as placas de Petri plásticas foram compradas em lojas de embalagens. Foram utilizados LEDs com máximo de emissão em 506 nm (verde, para a ERI) e $632 \mathrm{~nm}$ (vermelho, para o AM), facilmente encontrados em casas de produtos eletrônicos. Cada fonte de iluminação apresentava um conjunto de seis LEDs, cuja confecção segue descrita no Material Suplementar (Figura 1S).

$\mathrm{O}$ meio de cultura foi preparado em uma chapa de aquecimento (marca Kasvi). Os espectros de absorção e de fluorescência dos FS foram obtidos respectivamente em um espectrofotômetro Varian Cary-60 e um espectrofluorímetro Varian Cary-Eclipse. A irradiância absoluta dos LEDs foi avaliada num espectroradiômetro obtido pela Ocean Optics modelo USB2000+. Estes últimos equipamentos na realidade são opcionais, porém seu uso é desejável, pois auxiliam bastante o entendimento dos conceitos discutidos quantitativamente no trabalho.

Caso deseje, o professor pode optar pela utilização de meios de cultura não seletivos e próprios para o crescimento microbiano, como o Brain Heart Infusion (BHI) ou o Plate Count Agar (PCA), evitando assim impurezas procedentes do caldo de carne. Também é possível realizar os procedimentos utilizando corantes de grau analítico, para evitar a presença de excipientes e contaminantes.

\section{Métodos}

\section{Preparo das soluções de corantes}

As soluções dos corantes foram preparadas separadamente por meio da adição de uma gota de cada um dos compostos comerciais em $200 \mathrm{~mL}$ de água destilada. Dessa forma, ambos os FS chegaram à concentrações próximas a $6,0 \times 10^{-6} \mathrm{~mol} \mathrm{~L}^{-1}$. A concentração de cada FS foi determinada por meio do espectro de absorção de cada solução utilizando os valores de absortividade molar do AM $\varepsilon_{664 \mathrm{~nm}}=39,0 \times 10^{3} \mathrm{~L} \mathrm{~mol}^{-1} \mathrm{~cm}^{-1}$ e da ERI $\varepsilon_{532 \mathrm{~nm}}=96,6 \times 10^{3} \mathrm{~L} \mathrm{~mol}^{-1} \mathrm{~cm}^{-1}$ em água e solução tamponada $(\mathrm{pH} 7.4)$, respectivamente. ${ }^{24,34}$ Foram considerados os valores de absortividade molar das substâncias puras, uma vez que a proporção de outros compostos é consideravelmente menor não alterando assim o perfil espectrofotométrico dos corantes.

\section{Preparo do meio de cultura e distribuição em placas}

Para o preparo do meio de cultura sólido, foram adicionados 1,6 g de agar-agar e 1/4 de um cubo de caldo de carne $(2,4 \mathrm{~g})$ a $100 \mathrm{~mL}$ de água destilada. A mistura foi levada ao aquecimento e permaneceu em ebulição por cinco minutos. Após esse procedimento, $3 \mathrm{~mL}$ do meio de cultura foram transferidos para a placa e aguardou-se o processo de gelificação. Para evitar a contaminação do meio de cultura pelo ar na transferência para a placa de controle negativo, utilizou-se um fogareiro para que todo o processo fosse feito próximo à zona estéril da chama.

O meio de cultura para o controle positivo foi preparado como mencionado acima e a transferência para as placas ocorreu na ausência da chama. Nesse caso, após a solidificação do ágar, as placas foram contaminadas passando-se levemente o dedo indicador por toda a superfície do meio de cultura. Antes deste procedimento o responsável pela contaminação tocou/manuseou diversas maçanetas de portas do ambiente. $\mathrm{O}$ controle positivo 1 não recebeu nenhum tipo de tratamento. Já os controles 2 (sem AM) e 3 (sem ERI) foram iluminados a uma distância de $5 \mathrm{~cm}$ por 30 minutos com LEDs vermelhos $\left(\operatorname{LED}_{\text {verm }}\right)$ e verdes $\left(\operatorname{LED}_{\text {verd }}\right)$, respectivamente. A iluminação foi iniciada imediatamente após a contaminação das placas (Figura 2S).

Para os ensaios na presença dos fotossensibilizadores o meio de cultura e a contaminação foram feitos como descritos anteriormente. Nesses casos, antes da solidificação $2 \mathrm{~mL}$ da solução de corante foram adicionados. As amostras foram submetidas a 30 minutos de iluminação (ou não - controle escuro) com LED vermelho (AM, $\mathrm{LED}_{\text {verm }}$ ) ou verde (ERI, $\mathrm{LED}_{\text {verd }}$ ), nas mesmas condições já descritas.

Ao todo foram preparadas oito amostras, cujos detalhamentos encontram-se resumidos na Tabela 1.

\section{Análise qualitativa dos resultados}

Acompanhou-se o tempo de crescimento dos microrganismos, sendo que esse é diretamente influenciado pela temperatura e, portanto, por questões climáticas. Como o experimento descrito neste trabalho foi realizado durante o período de inverno, todas as imagens foram coletadas após dez dias da contaminação/tratamento das placas.

\section{Medida da irradiância dos LEDs e cálculo do número de fótons absorvidos}

Os cálculos de potência absorvida (PAbs) e número de fótons absorvidos (NAbs) foram realizados tomando-se a potência da luz incidente (PEm) e o espectro de absorção do FS seguindo a metodologia proposta por Rabello e colaboradores. ${ }^{38}$ Uma descrição detalhada dessa metodologia segue descrita no Material Suplementar. Os cálculos sugeridos, apesar de auxiliarem uma maior compreensão quantitativa do experimento, não são totalmente necessários, cabendo ao professor esta decisão.

\section{RESULTADOS E DISCUSSÃO}

O controle microbiano consiste em uma série de procedimentos que impedem a proliferação de microrganismos existentes em determinados utensílios ou ambientes. Nesse sentido, o controle negativo é uma amostra importante em análises microbiológicas para demonstrar

Tabela 1. Conjunto de amostras preparadas

\begin{tabular}{|c|c|c|c|c|c|}
\hline Amostras & Contaminação & $\mathrm{AM}^{\mathrm{a}}$ & $\mathrm{ERI}^{\mathrm{a}}$ & Iluminação LED verm $_{\text {ver }}$ & Iluminação LED $_{\text {verd }}$ \\
\hline Controle Negativo & - & - & - & - & - \\
\hline Controle Positivo 1 & $\mathrm{X}$ & - & - & - & - \\
\hline Controle Positivo 2 & $X$ & - & - & $\mathrm{X}$ & - \\
\hline Controle Positivo 3 & $\mathrm{X}$ & - & - & - & $\mathrm{X}$ \\
\hline Controle AM escuro & $\mathrm{X}$ & $\mathrm{X}$ & - & - & - \\
\hline $\mathrm{AM} / \mathrm{LED}_{\text {verm }}$ & $\mathrm{X}$ & $\mathrm{X}$ & - & $\mathrm{X}$ & - \\
\hline Controle ERI escuro & $\mathrm{X}$ & - & $\mathrm{X}$ & - & - \\
\hline $\mathrm{ERI} / \mathrm{LED}_{\text {verd }}$ & $\mathrm{X}$ & - & $\mathrm{X}$ & - & $\mathrm{X}$ \\
\hline
\end{tabular}

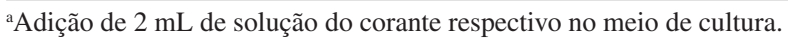


que o crescimento microbiano ocorre exclusivamente devido à contaminação intencional do meio de cultura. Para evitar que o meio de cultura seja contaminado pelo contato com o ar na transferência do béquer para a placa, esse processo foi realizado próximo a uma chama. Este método de transferência próximo à zona estéril da chama e a ausência de qualquer outro tipo intencional de contaminação resultou em uma placa sem qualquer crescimento microbiano visível, como pode ser observado na Figura $2 \mathrm{~A} .{ }^{43}$

Já o controle positivo (Figura 2B), mostra como ocorre a contaminação, sem que seja realizado qualquer tratamento no intuito de controlar o crescimento microbiano.
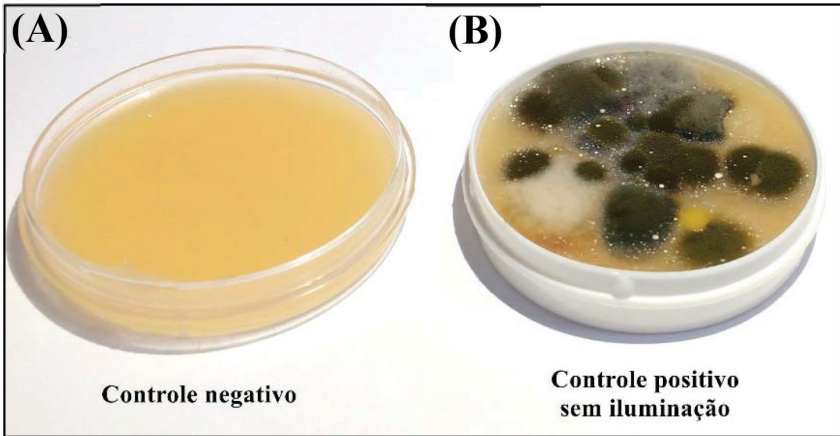

Figura 2. Placas de (A) controle negativo e (B) controle positivo 1 (sem iluminação)

Como citado anteriormente na seção experimental, antes de contaminar as placas, o responsável por esta etapa abriu/fechou diversas portas, representando assim a contaminação de maçanetas de uso comum. A literatura já tem reportado a presença da bactéria Gram-positiva Staphylococcus aureus em maçanetas de hospitais e destacado a extrema importância de procedimentos que minimizem o contágio, como higienização das mãos e das superfícies frequentemente manuseadas. ${ }^{44,45}$

Algumas bactérias, como a Escherichia coli, classificadas como Gram-negativas, dificilmente são eliminadas por ativos antimicrobianos. Isso pode ser explicado pela presença de uma membrana externa adicional complexa incluindo duas bicamadas lipídicas. ${ }^{43}$ $\mathrm{Na}$ presença de qualquer ameaça, a membrana externa atua como barreira de proteção física e funcional, inibindo a penetração de antibióticos e acionando mecanismos de defesa. Essas bactérias também são encontradas como contaminantes em maçanetas ${ }^{46}$ e trabalhos presentes na literatura tem reportado efeitos promissores da TFDA na inibição desta classe de microrganismos. ${ }^{47,48}$ Já bactérias como a Staphylococcus aureus são mais facilmente atingidas pela atividade fotodinâmica, visto que não possuem membrana de proteção externa e sua camada de peptideoglicano fica exposta ao ataque de espécies citotóxicas. . $^{30,37,47}$

Além de bactérias, o controle positivo demonstrou um grande crescimento de espécies de fungos filamentosos. Os fungos também denotam grande preocupação da área médica. Estes microrganismos apresentam elevada resistência devido à presença de uma membrana nuclear que representa uma barreira adicional à penetração de medicamentos. No entanto, vários estudos tem demonstrado a atividade fotodinâmica do fenotiazínico Azul de Metileno capaz de causar danos estruturais na membrana fúngica. ${ }^{48-50}$

Como descrito anteriormente, é conhecido que mesmo mantido no escuro o Azul de metileno possui atividade antimicrobiana. Essa característica do corante fenotiazínico é utilizada, por exemplo, em meios de cultura seletivos à bactérias gram-negativas, como o ágar eosina-azul de metileno. Isso ocorre pois o AM é capaz de inibir o crescimento de bactérias gram-positivas. ${ }^{43}$
Os testes realizados com este corante, no entanto, indicaram que a atividade do AM no escuro (Figura 3A) é baixa quando comparada a ação fotodinâmica ilustrada pela placa que foi iluminada por LEDs vermelhos (30 minutos de iluminação, Figura 3B). Nota-se que a iluminação acarretou em efeitos fotodinâmicos pronunciados, dado o mínimo nível de crescimento microbiano observado. Estes resultados demonstram a potencialidade fotodinâmica em danos celulares nas mais diversas espécies de microrganismos.

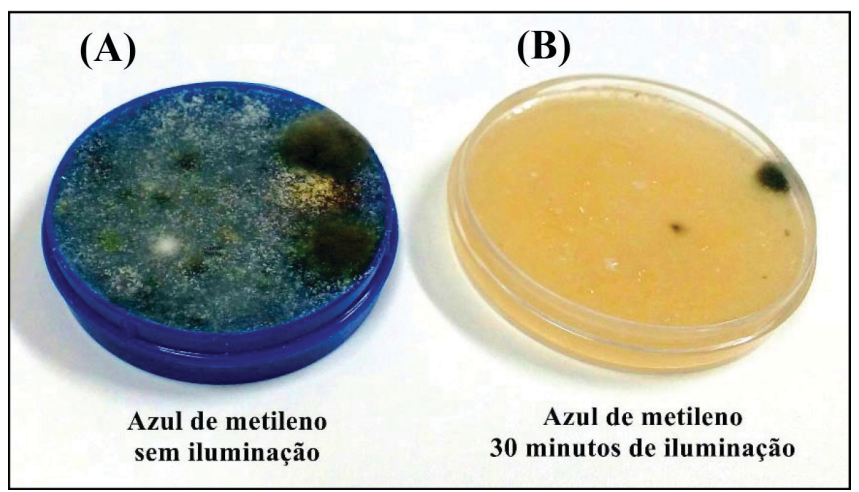

Figura 3. Placas de crescimento microbiano em meio de cultura contendo Azul de Metileno: (A) sem iluminação e (B) iluminada por trinta minutos (imagens obtidas após 10 dias da iluminação)

Nos experimentos realizados, a Eritrosina B mostrou-se também extremamente eficaz na inibição do crescimento microbiano, como ilustrado na comparação entre as Figuras 4A e 4B, na ausência e presença de luz, respectivamente. Comparada ao controle positivo, a amostra contendo Eritrosina B, mesmo sem iluminação, apresentou diminuição do crescimento microbiano. Contudo, a placa exposta à iluminação por trinta minutos com LEDs verdes não apresentava qualquer sinal de crescimento de microrganismos, mesmo após dez dias.

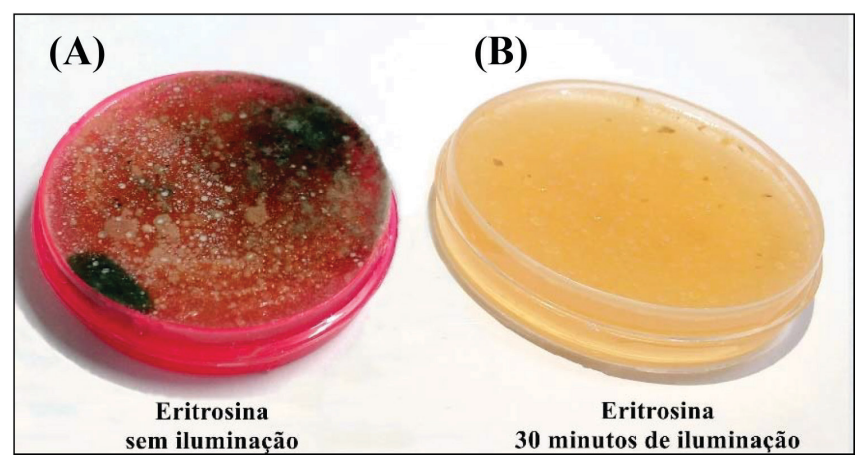

Figura 4. Placas de crescimento microbiano em meio de cultura contendo Eritrosina: (A) sem iluminação e (B) iluminada por 30 minutos (imagens obtidas após 10 dias da iluminação)

Comparando a ação fotodinâmica de ambos os corantes, nota-se que a ERI inibiu completamente o crescimento microbiológico, enquanto que para o AM um pouco de fungos filamentosos ainda podem ser observados na amostra iluminada (Figura 3B). Esse fato poderia ser explicado devido à diferença de potência dos LEDs, maior absorção de fótons ou ainda maior rendimento quântico de oxigênio singleto por parte do sistema com a Eritrosina, uma vez que a concentração dos FS é similar.

A discussão acima sob o ponto de vista quantitativo será dada para dirimir essas dúvidas. Foi necessário então, entre outros, realizar o mesmo experimento avaliando se apenas a iluminação seria uma causa de inativação dos microrganismos (Figura 5). 


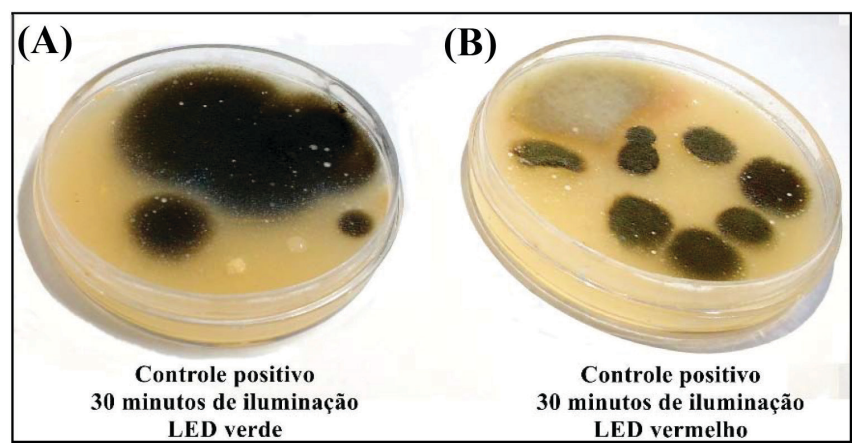

Figura 5. Placas de: (A) controle positivo 2 iluminada pelo sistema $L E D_{\text {verd }}$ com $\lambda_{\max }=506 \mathrm{~nm}$ e $(B)$ controle positivo 3 iluminada pelo sistema $L E D_{\text {verm }}$ $\operatorname{com} \lambda_{\max }=632 \mathrm{~nm}$

A análise da Figura 5 mostra que na ausência do corante, a iluminação não é capaz de impedir o crescimento de bactérias e fungos sobre o meio de cultura. Isso confirma que a inibição vista nos casos anteriores ocorre necessariamente devido a processos fotodinâmicos dos corantes. Esses experimentos são somente de controles.

Vários fatores podem estar atuando independentemente ou sinergicamente na atividade fotodinâmica dos FS utilizados, podendo justificar as diferenças observadas. Primeiramente, apesar da concentração empregada para ambos FS ser muito semelhante, é preciso salientar que a absortividade molar da ERI é significativamente superior à do AM, o que afeta o número de fótons absorvidos. Outro fator atrelado

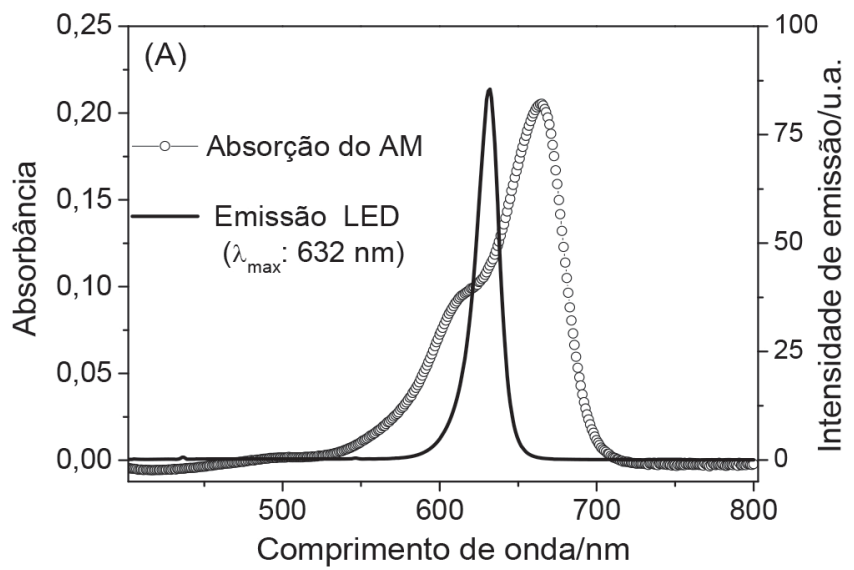

à absorção de luz que pode estar corroborando com o maior efeito fotodinâmico apresentado pela ERI é a sua elevada eficiência na geração de ${ }^{1} \mathrm{O}_{2}$, relativamente superior à do $\mathrm{AM},\left(\mathrm{AM}: \Phi_{\Delta}{ }^{1} \mathrm{O}_{2}=0,50^{25} \mathrm{e}\right.$ ERI: $\left.\Phi_{\Delta}{ }^{1} \mathrm{O}_{2}=0,63^{51}\right)$. Esse é sem dúvida um importante parâmetro relativo à morte dos microrganismos. Contudo, a geração de ${ }^{1} \mathrm{O}_{2}$ depende da quantidade de luz que o FS está recebendo por unidade de tempo, isto é, a dose de luz/irradiância por tempo. No entanto, a determinação dos valores de irradiância para cada fonte de luz utilizada (Tabela 2) demonstrou que o sistema de LEDs vermelhos apresenta irradiância superior à do sistema verde, o que vai contra os resultados observados. Isto demonstra que para a eficiência fotodinâmica existem vários fatores a serem considerados.

Dentro desses fatores, um dos mais determinantes é o nível de sobreposição espectral (área) entre cada FS e sua respectiva fonte de excitação. As sobreposições são apresentadas na Figura 6.

Nota-se, na Figura 6, que tanto no sistema AM/LED ${ }_{\text {verm }}$ como no sistema ERI/LED ${ }_{\text {verd }}$ houve significativa sobreposição espectral. No entanto, uma análise visual qualitativa indica que apesar da maior irradiância da fonte de luz vermelha, a sobreposição foi

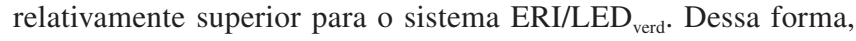
aliado a uma maior absortividade da ERI (2,5 vezes maior), uma maior quantidade de fótons são absorvidos por parte do corante xantênico em detrimento do fenotiazínico. Com intuito de analisar os dados de maneira quantitativa, foi então calculada a potência total absorvida em cada sistema, conforme representado na Figura 7. Os dados foram obtidos seguindo-se o descrito por Rabello e colaboradores. $^{38}$

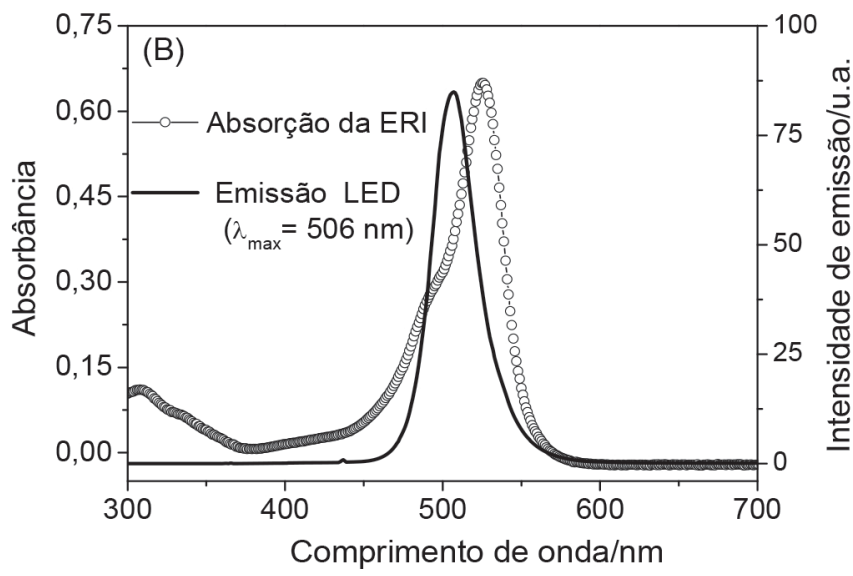

Figura 6. Sobreposição dos espectros de emissão relativa dos LEDs e da absorção eletrônica dos corantes em água: (A) AM/LED $D_{\text {verm }}$ (B) ERI/LED ${ }_{\text {verd }}$ solução tamponada $(\mathrm{pH} \mathrm{7,4)}$
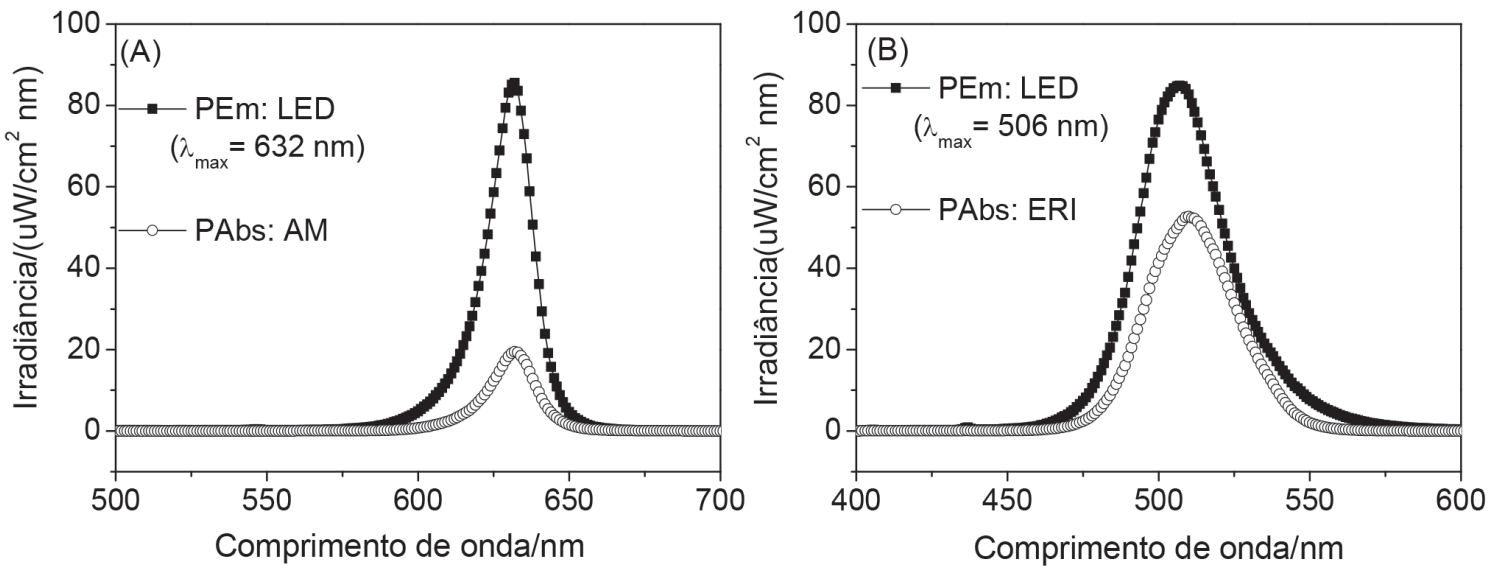

Figura 7. Representação espectral da potência de luz total (PEm) emitida pelos diodos emissores de luz (LEDs) e potência absorvida pelos FS (PAbs): (A) $A M$ e (B) ERI 
Tabela 2. Valores de irradiância das fontes de luz, porcentagem da potência total absorvida pelos FS (PAbs \%) e fótons absorvidos (NAbs) por cada sistema utilizado

\begin{tabular}{|c|c|c|c|}
\hline Sistema & Irradiância $\left(\mathrm{mW} / \mathrm{cm}^{2}\right)$ & PAbs $(\%)$ & NAbs $\left(10^{-8} \mathrm{~mol}\right)$ \\
\hline $\mathrm{AM} / \mathrm{LED}_{\text {verm }}\left(\lambda_{\max }=632 \mathrm{~nm}\right)$ & 1,94 & 21,8 & 1,0 \\
\hline ERI $/$ LED $_{\text {verd }}\left(\lambda_{\max }=506 \mathrm{~nm}\right)$ & 1,42 & 58,0 & 4,8 \\
\hline
\end{tabular}

A análise da Figura 7 mostra que a PAbs pelo sistema AM/LED ${ }_{\text {verm }}$ é bastante inferior à PAbs pelo sistema ERI/LED ${ }_{\text {verd. }}$ A partir dos valores de PAbs foi possível estimar a porcentagem de luz absorvida por cada FS e o respectivo número de fótons realmente absorvidos (NAbs). Os dados obtidos encontram-se na Tabela 2.

Diante dos resultados apresentados na Tabela 2, apesar da maior irradiância do $L_{E D}$ verm em relação ao $L E D_{\text {verd }}$, pode-se inferir que de fato a ERI teve a dose de luz absorvida significativamente superior à do AM (praticamente 5x). Dessa forma, o maior efeito fotodinâmico da ERI pode ser plenamente justificado pela associação entre a maior dose de luz absorvida atrelada à maior sobreposição espectral e elevados valores de absortividade molar, bem como a maior geração de ${ }^{1} \mathrm{O}_{2}$ previamente reportados na literatura. ${ }^{33,34}$ Ainda assim, os efeitos observados para o AM foram bastante satisfatórios, sendo ambos FS fortes candidatos a aplicações biomédicas no controle de microrganismos. Obviamente no aspecto bioquímico se deve ainda considerar a importante interação entre os corantes (catiônico AM e o dianiônico ERI) com os microrganismos investigados, o que no trabalho em questão não foi o foco.

Acrescenta-se que no intuito de formar amplos conceitos aos acadêmicos, a totalidade da prática proposta é condizente. No entanto, devido às dificuldades de terem-se os equipamentos e materiais adequados aliados a questões de tempo de aulas experimentais, é factível uma redução nos experimentos. Por exemplo, a ausência de um medidor de potência/irradiância para os LEDs e/ou ausência de um espectrofluorímetro para a determinação do espectro de emissão dos LEDs impedem a análise quantitativa dos resultados. Entretanto torna-se importante ter em mente as propriedades físicas da luz e seus processos de absorção o que permite executar qualitativamente o experimento, bem como efetuar-se a escolha adequada do par FS/ LED. No caso do AM, a cor azul implica em não absorção da luz azul, cor enxergada, e portanto absorção de luz na região complementar conforme exemplificado pelo disco de Newton (Figura 8), ou seja, absorção de luz na região do laranja/vermelho (no caso usou-se $\left.\mathrm{LED}_{\text {verm }}\right)$. No caso da ERI, sua coloração rosa implica em absorção de luz na região próxima a amarelo/verde $\left(\mathrm{LED}_{\text {verd }}\right)$ conforme novamente justificado pela Figura 8.

Outra simplificação possível é o uso de apenas um corante fotossensibilizador. Neste caso, somente seria demonstrada a atividade fotodinâmica FS/LED, sem a necessidade de cálculos de fótons efetivamente absorvidos. Alternativamente, pode-se variar a concentração do corante ou a distância de iluminação. Ainda é possível avaliar a

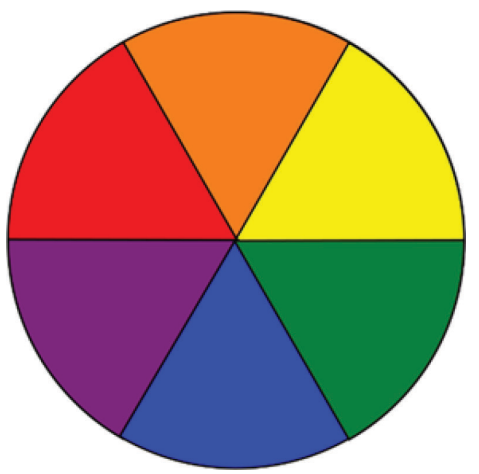

Figura 8. Disco de Newton indicando as cores complementares atividade de outros compostos, como por exemplo da clorofila $\left(\Phi_{\Delta}{ }^{1} \mathrm{O}_{2}\right.$ $=0,44),{ }^{52}$ cuja cor verde implica em absorção de luz vermelha (Figura 8) e é facilmente extraída de folhas de espinafre. Com relação à fonte de luz, várias alternativas são possíveis, por exemplo, lanterna de celular ou mesmo a luz solar com os cuidados necessários para que o meio de cultura não seja liquefeito ou contaminado não intencionalmente e inviabilize o experimento.

Por outro lado, para cursos de farmácia, bioquímica e biologia também são possíveis modificações tomando-se bactérias e fungos previamente selecionados, permitindo uma abordagem bastante ampla seguida de discussões sobre a estruturação de suas membranas que atuam como barreiras de proteção.

\section{CONCLUSÕES}

No experimento descrito neste trabalho evidencia-se claramente a atividade fotodinâmica de dois corantes comerciais de fácil aquisição. A Eritrosina B apresentou uma inibição maior que o Azul de Metileno. Essa maior eficiência mostrou-se associada ao rendimento quântico de oxigênio singleto e a um maior número de fótons efetivamente absorvidos por esse corante, uma vez que a sobreposição espectral da $E R I / L E D_{\text {verd }}$ foi maior do que a do par $A M / L D_{\text {verm }}$.

Desse modo, discussões acerca de reações fotoquímicas em cursos de química ou mesmo sobre controle microbiológico em aulas de biologia podem se tornar mais interessantes e contextualizadas. $\mathrm{O}$ professor pode se utilizar deste experimento para introduzir discussões sobre o uso inadequado de antibióticos, técnicas de tratamento de câncer ou de doenças microbianas, conceitos teóricos de espectroscopia e fotoquímica, entre outros tantos assuntos englobados através desta atividade experimental.

Por fim, este experimento, com as devidas simplificações e adaptações, pode ser incluído nas disciplinas práticas dos cursos de graduação em Química, ou ainda para aulas interdisciplinares de Farmácia, Bioquímica e Biologia.

\section{MATERIAL SUPLEMENTAR}

No material suplementar, disponível em http://quimicanova.sbq. org.br em arquivo PDF de livre acesso, encontra-se detalhado o preparo do sistema LED utilizado (Figura 1S), assim como a iluminação das placas (Figura 2S).

Acrescentam-se os procedimentos detalhados da medida da irradiância dos LEDs e cálculo do número de fótons absorvidos realmente pelos corantes (NAbs).

\section{AGRADECIMENTOS}

Os autores agradecem à UGF/SETI-PR, à Fundação Araucária/ SETI-PR, ao CNPq e à CAPES pelo suporte financeiro.

\section{REFERÊNCIAS}

1. Abdel-Kader, M. H. In Photodynamic Therapy - from theory to application; Abdel-Kader, M. H., ed.; Springer: Berlin, 2014; pp. 3-22.

2. Figueiras, C. A. L.; Quim. Nova Esc. 1996, 22.

3. Machado, A. E. da H.; Quim. Nova 2000, 23, 237. 
4. Simplicio, F. I.; Maionchi, F.; Hioka, N.; Quim. Nova 2002, 25, 801.

5. MacDonald, I. J.; Dougherty, T. J.; J. Porphyrins Phthalocyanines 2001, $5,105$.

6. Celli, J. P.; Spring, B. Q.; Rizvi, I.; Evans, C. L.; Samkoe, K. S.; Verma, S.; Pogue, B. W.; Hasan, T.; Chem. Rev. 2010, 110, 2795.

7. Dougherty, T. J.; Gomer, C. J.; Henderson, B. W.; Jori, G.; Kessel, D.; Korbelik, M.; Moan, J.; Peng, Q.; J. Natl. Cancer Inst. 1998, 90, 889.

8. Jori, G.; Fabris, C.; Soncin, M.; Ferro, S.; Coppellotti, O.; Dei, D.; Fantetti, L.; Chiti, G.; Roncucci, G.; Lasers Surg. Med. 2006, 38, 468.

9. Wainwright, M.; Color. Technol. 2017, 133, 3.

10. Wainwright, M.; Maisch, T.; Nonell, S.; Plaetzer, K.; Almeida, A.; Tegos, G. P.; Hamblin, M. R.; Lancet Infect. Dis. 2017, 17, 49.

11. Wainwright, M.; Int. J. Antimicrob. Agents 2014, 44, 26.

12. Perussi, J. R.; Quim. Nova 2007, 30, 988.

13. Wainwright, M.; Mohr, H.; Walker, W. H.; J. Photochem. Photobiol., B 2007, 86, 45 .

14. Bagnato, V. S.; Kurachi, C.; Ferreira, J.; Marcassa, L. G.; Sibata, C. H.; Allison, R. R.; Photodiagn. Photodyn. Ther. 2005, 2, 107.

15. Eduardo, C. de P.; Bello-Silva, M. S.; Ramalho, K. M.; Lee, E. M. R.; Aranha, A. C. C.; Rev. Assoc. Paul. Cir. Dent. 2015, 69, 226.

16. Nagata, J. Y.; Hioka, N.; Kimura, E.; Batistela, V. R.; Terada, R. S. S.; Graciano, A. X.; Baesso, M. L.; Hayacibara, M. F.; Photodiagn. Photodyn. Ther. 2012, 9, 122.

17. Marotti, J.; Tortamano, P.; Cai, S.; Ribeiro, M. S.; Franco, J. E. M.; de Campos, T. T.; Lasers Med. Sci. 2013, 28, 303.

18. Issa, M. C. A.; Manela-Azulay, M.; An. Bras. Dermatol. 2010, 85, 501.

19. de Melo, W. C.; Avci, P.; de Oliveira, M. N.; Gupta, A.; Vecchio, D.; Sadasivam, M.; Rakkiyappan, C.; Huang, Y.-Y.; Yin, R.; Perussi, L. R.; Tegos, G. P.; Perussi, J. R.; Dai, T.; Hamblin, M. R.; Expert Rev. Anti. Infect. Ther. 2013, 11, 669.

20. Rosa, L. P.; Silva, F. C.; Vieira, R. L.; Tanajura, B. R.; Gusmão, A. G. da S.; de Oliveira, J. M.; dos Santos, N. A. C.; Bagnato, V. S.; Photodiagn. Photodyn. Ther. 2017, 19, 235.

21. Weijer, R.; Broekgaarden, M.; Kos, M.; van Vught, R.; Rauws, E. A. J.; Breukink, E.; van Gulik, T. M.; Storm, G.; Heger, M.; J. Photochem. Photobiol., C 2015, 23, 103.

22. Schirmer, R. H.; Adler, H.; Pickhardt, M.; Mandelkow, E.; Neurobiol. Aging 2011, 32, 7.

23. Oz, M.; Lorke, D. E.; Petroianu, G. A.; Biochem. Pharmacol. 2009, 78, 927.

24. Santin, L. R. R.; Santos, S. C. dos; Novo, D. L. R.; Bianchini, D.; Gerola, A. P.; Braga, G.; Caetano, W.; Moreira, L. M.; Bastos, E. L.; Romani, A. P.; de Oliveira, H. P. M.; Dyes Pigm. 2015, 119, 12.

25. Tardivo, J. P.; Giglio, A. del; Oliveira, C. S.; Gabrielli, D. S.; Junqueira, H. C.; Tada, D. B.; Severino, D.; Turchiello, R. de F.; Baptista, M. S.; Photodiagn. Photodyn. Ther. 2005, 2, 175.

26. Tardivo, J. P.; Del Giglio, A.; Paschoal, L.; Ito, A.; Baptista, M.; Photodiagn. Photodyn. Ther. 2004, 1, 345.

27. Lee, Y. S.; Wurster, R. D.; Cancer Lett. 1995, 88, 141.

28. Trindade, G. S.; Farias, S. L. A.; Rumjanek, V. M.; Capella, M. A. M.; Cancer Lett. 2000, 151, 161.

29. Wainwright, M.; Phoenix, D. a; Gaskell, M.; Marshall, B.; J. Antimicrob. Chemother. 1999, 44, 823.
30. Wainwright, M. Y.; Phoenix, D. A.; Laycock, S. L.; Wareing, D. R. A.; Wright, P. A.; FEMS Microbiol Lett. 1998, 160, 177.

31. Peloi, L. S.; Biondo, C. E. G.; Kimura, E.; Politi, M. J.; Lonardoni, M. V. C.; Aristides, S. M. A.; Dorea, R. C. C.; Hioka, N.; Silveira, T. G. V.; Exp. Parasitol. 2011, 128, 353.

32. Tardivo, J. P.; Serrano, R.; Zimmermann, L. M.; Diabet. Foot Ankle 2017, 8,1 .

33. Gandin, E.; Lion, Y.; Van de Vorst, A.; Photochem. Photobiol. 1983, 37, 271.

34. Batistela, V. R.; Pellosi, D. S.; De Souza, F. D.; Da Costa, W. F.; De Oliveira Santin, S. M.; De Souza, V. R.; Caetano, W.; De Oliveira, H. P. M.; Scarminio, I. S.; Hioka, N.; Spectrochim. Acta, Part A 2011, 79, 889.

35. Fracalossi, C.; Yuri, J.; Silva, D.; Sano, R.; Terada, S.; Hioka, N.; Luciano, M.; Sato, F.; Luiz, P.; Caetano, W.; Fujimaki, M.; Photodiagn. Photodyn. Ther. 2016, 15, 127.

36. Wood, S.; Metcalf, D.; Devine, D.; Robinson, C.; J. Antimicrob. Chemother. 2006, 57, 680.

37. Yassunaka, N. N.; de Freitas, C. F.; Rabello, B. R.; Santos, P. R.; Caetano, W.; Hioka, N.; Nakamura, T. U.; de Abreu Filho, B. A.; Mikcha, J. M. G.; Curr. Microbiol. 2015, 71, 243.

38. Rabello, B. R.; Gerola, A. P.; Pellosi, D. S.; Tessaro, A. L.; Aparício, J. L.; Caetano, W.; Hioka, N.; J. Photochem. Photobiol., A 2012, 238, 53.

39. Maluta, J. R.; Quim. Nova 2014, 37, 1244.

40. Rapp, T. L.; Phillips, S. R.; Dmochowski, I. J.; J. Chem. Educ. 2016, 93, 2101.

41. Prabpal, J.; Vilaivan, T.; Praneenararat, T.; J. Chem. Educ. 2017, 94, 1137.

42. Atkins, P.; de Paula, J.; Físico-Química, 9ª ed., LTC Editora: São Paulo, 2012.

43. Madigan, M. T.; Microbiologia de Brock, 14ª ed.; Artmed: São Paulo, 2016.

44. Silva, S. A.; Deuschle, R. A. N.; Mion, C. D. C. M.; Revista Saúde (Santa Maria) 2012, 38, 129.

45. Oie, S.; Hosokawa, I.; Kamiya, A.; J. Hosp. Infect. 2002, 51, 140.

46. Bashir, S. F.; Muhammad, H.; Sani, N. M.; Kawo, A. H.; J. Pharm. Biol. Sci. 2016, 11, 53.

47. Monteiro, J. S. C.; Oliveira, S. C. P. S. De; Santos, G. M. P.; José, F.; Sampaio, P.; Guilherme, L.; Soares, P.; Pinheiro, A. L. B.; Lasers Med. Sci. 2017, 29.

48. Peloi, L. S.; Soares, R. R. S.; Biondo, C. E. G.; Souza, V. R.; Hioka, N.; Kimura, E.; J. Biosci. 2008, 33, 231.

49. Teichert, M. C.; Jones, B. A.; Usacheva, M. N.; Biel, M. A.; Oral Surg. Oral Med. Oral Pathol. 2002.

50. Baltazar, L. M.; Ray, A.; Santos, D. A.; Cisalpino, P. S.; Friedman, A. J.; Nosanchuk, J. D.; Front. Microbiol. 2015, 6, 1.

51. Pellosi, D. S.; Estevão, B. M.; Freitas, C. F.; Tsubone, T. M.; Caetano, W.; Hioka, N.; Dyes Pigm. 2013, 99, 705.

52. Gerola, A. P.; Semensato, J.; Pellosi, D. S.; Batistela, V. R.; Rabello, B. R.; Hioka, N.; Caetano, W.; J. Photochem. Photobiol., A 2012, 232, 14. 